\title{
Random percolation as a gauge theory
}

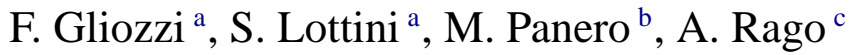 \\ a Dipartimento di Fisica Teorica, Università di Torino and INFN, Sezione di Torino, \\ via P. Giuria, 1, I-10125 Torino, Italy \\ b Dublin Institute for Advanced Studies, 10 Burlington Road, Dublin 4, Ireland \\ ${ }^{\mathrm{c}}$ Dipartimento di Fisica, Università di Milano and INFN, Sezione di Milano, \\ Via Celoria, 16, I-20133 Milano, Italy
}

Received 23 February 2005; accepted 15 April 2005

Available online 10 May 2005

\begin{abstract}
Three-dimensional bond or site percolation theory on a lattice can be interpreted as a gauge theory in which the Wilson loops are viewed as counters of topological linking with random clusters. Beyond the percolation threshold large Wilson loops decay with an area law and show the universal shape effects due to flux tube quantum fluctuations like in ordinary confining gauge theories. Wilson loop correlators define a non-trivial spectrum of physical states of increasing mass and spin, like the glueballs of ordinary gauge theory. The crumbling of the percolating cluster when the length of one periodic direction decreases below a critical threshold accounts for the finite temperature deconfinement, which belongs to 2D percolation universality class.
\end{abstract}

(C) 2005 Elsevier B.V. All rights reserved.

PACS: 11.15.Ha; 05.50.+q; 05.70.Jk; 05.70.Lq

\section{Introduction}

Percolation is a purely geometrical phenomenon which in many respects resembles a continuous thermal phase transition. The theoretical description of the percolation processes is conventionally given in terms of the cluster sizes [1], and most of the uni-

E-mail addresses: gliozzi@to.infn.it (F. Gliozzi), lottini@to.infn.it (S. Lottini), panero@stp.dias.ie (M. Panero), antonio.rago@mi.infn.it (A. Rago). 
versal scalings deal with size distribution of clusters. The point of view which is taken in this paper is different. We focus on topological entanglement of random clusters and use it to describe how percolation theory in three dimensions can be viewed as a full-fledged gauge theory.

Though the gauge group of the theory in question is trivial (it is the $q \rightarrow 1$ limit of the symmetric group $S_{q}$ ), the occurrence of a confining phase yields some new hints on the mechanisms of quark confinement of more general theories.

Transcribing percolation in terms of gauge theory has also some important consequences for percolation itself. In three-dimensional systems there are almost no exact results whatsoever, but in gauge theories we have a number of well-verified conjectures that can be translated into the language of percolation. In this way we shall, for instance, relate certain linking properties of the closed paths within a percolating cluster to the universal quantum fluctuations of the chromoelectric flux tube joining a quark pair in the confining phase of whatever gauge theory.

An unsuspected property of random percolation which emerges from this new viewpoint is that one can build up new classes of correlators which define, through their exponential decay, a variety of different correlation lengths $\xi_{1}>\xi_{2}>\cdots$. Their inverse $1 / \xi_{1}<$ $1 / \xi_{2}<\cdots$ form, in the gauge theory language, the mass spectrum of the model, which turns out to be composed by a (possibly infinite) tower of physical states of increasing mass and spin-the glue-balls of the corresponding gauge theory. Their mass ratios near the percolation point define a totally new set of critical amplitude ratios belonging to the universality class of $3 \mathrm{D}$ random percolation.

Another piece of useful information comes from considering percolation in a slab which is infinite in two dimensions, but of finite length $\ell$ and periodic in the remaining direction. The associated gauge model describes a system at finite temperature $T=1 / \ell$. This transition is accurately described by the universality class of two-dimensional random percolation, but the corresponding deconfining temperature $T_{c}$ may be used to define a new critical amplitude of the three-dimensional system.

We test the universality of this new set of critical amplitudes by performing large scale numerical experiments in three different kinds of lattices: the simple cubic (SC) with bond or site percolation and the body centered cubic lattice (BCC) with bond percolation. The numerical implementation of these systems is straightforward in comparison with simulations of ordinary gauge models: no Markovian process is needed and there are neither thermalization problems nor critical slowing down. Preliminary results have been presented in [2].

The organization of the paper is as follows. In Section 2 we define a new class of observables of the percolation theory to be identified with the Wilson loops of the corresponding gauge theory. In Section 3 we describe a method to study the linking properties of the random clusters which is at the heart of our analysis. Section 4 is devoted to the comparison of confinement mechanisms and in Section 5 we extract the string tension (the gauge theory analogue of the surface tension) and show the relevance of the universal terms generated by quantum fluctuations of an underlying effective string. In Section 6 we study the plaquette correlators in order to find the low-lying states of the spectrum. Section 7 is devoted to the transcription of the percolation on a slab into a gauge system at finite temperature and Sec- 
tion 8 discusses the universality class of the finite temperature deconfinement transition. Finally in Section 9 we draw some concluding remarks.

\section{Observables}

The most basic observables of any gauge theory are the Wilson loops. These are operators which assign to each pair $(C, \gamma)$ formed by an arbitrary gauge configuration $C$ and any closed path $\gamma$ of the space a suitably defined complex number $W_{\gamma}(C)$. Their importance stems from the fact that they serve as order parameters for confinement. The confining phase is expected to show up in an area law for the vacuum expectation value of large Wilson loops. The area law means that if $\gamma$ is scaled up, keeping its shape fixed and increasing its encircled minimal area $A$, then $\left\langle W_{\gamma}\right\rangle$ vanishes exponentially with $A$ :

$$
\left\langle W_{\gamma}\right\rangle \propto e^{-\sigma A},
$$

where the area coefficient $\sigma$, called string tension, is the fundamental quantity of whatever confining gauge theory.

One can define similar observables in the framework of random percolation. In this context the configurations are generated simply by occupying each site or bond on an initially empty 3D lattice $\Lambda$ with independent probability $p$. Two sites are considered neighbours if they share one bond. The resulting configuration is a graph $G$ drawn on the lattice, composed by the occupied bonds (bond percolation problem) or by the bonds joining occupied neighbour sites (site percolation problem). The connected components of $G$ are the clusters of the configuration. We choose as $\gamma$ 's the closed paths of the dual lattice $\tilde{\Lambda}$. The value $W_{\gamma}(G)$ measures the topological entanglement between $\gamma$ and $G$. More precisely we apply the following rule

(1) $W_{\gamma}(G)=1$ if no cluster of $G$ is linked to $\gamma$;

(2) $W_{\gamma}(G)=0$ otherwise.

This definition did not come out of the blue. Starting from the Fortuin-Kasteleyn random cluster representation of the 3D Ising model [3] combined with the Kramers-Wannier duality [4], it is easy to express the Wilson loop $W_{\gamma}$ belonging to a $\mathbb{Z}_{2}$ gauge system in terms of the winding numbers modulo 2 of the Fortuin-Kasteleyn clusters across the $\gamma$ loop [5]. Generalizing this result to $q$-state Potts model one is led to the above rule in the case of non-integer $q$. A recently developed algorithm could even allow to evaluate explicitly these quantities for real $q>0$ [6]. We extend the above recipe to any percolating system, owing to the fact that bond percolation can be viewed as the $q \rightarrow 1$ limit of the $q$-state Potts model.

The removal of an occupied bond $b$ from a graph $G$ can lead to two different issues. If the number $c(G)$ of clusters is kept invariant, then $b$ is necessarily a step of a closed path (or loop) of $G$, whereas if $c(G)$ increases by one, $b$ cannot lie in a loop and is called a bridge (see Fig. 1). Clearly only the former bonds contribute to $W_{\gamma}(G)$. If two graphs $G$ and $G^{\prime}$ have the same loops and differ only in the bridges, then they yield the same value 


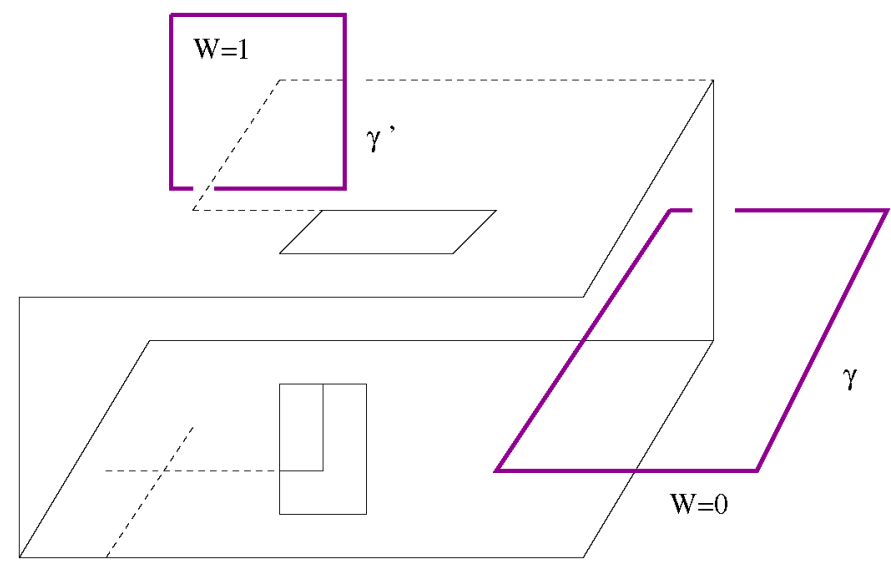

Fig. 1. The closed thick lines $\gamma$ and $\gamma^{\prime}$ represent Wilson loops. The dashed lines are bridges of the cluster. The other solid lines are closed paths of the cluster. $\gamma$ is linked to the cluster, while $\gamma^{\prime}$ is unlinked.

of $W_{\gamma}$ for any $\gamma$. In more technical terms we may write the double implication

$$
G \cup G^{\prime}-G \cap G^{\prime} \text { is a tree } \Leftrightarrow W_{\gamma}(G)=W_{\gamma}\left(G^{\prime}\right), \forall \gamma \subset \tilde{\Lambda},
$$

hence the transformation $G \rightarrow G^{\prime}$ has some resemblance to a gauge transformation.

Note that the connected correlator among occupied bonds is exactly zero by construction, but this is not an invariant quantity under the above $G \rightarrow G^{\prime}$ transformation. Cutting all bridges of $G$ yields the maximal invariant subset $B_{G}$ of $G$, made by bonds belonging to some loop. Of course we have $W_{\gamma}(G)=W_{\gamma}\left(B_{G}\right)$ for any $\gamma$. The connected correlator among bonds belonging to $B_{G}$ is by no means trivial and is directly related to the connected correlator of the plaquette, i.e., the Wilson loop $W_{\square}$ associated to the smallest loop $\square \subset \tilde{\Lambda}$, because $W_{\square}=0$ if and only if the bond dual to $\square$ belongs to $B_{G}$. In Section 6 we shall use such a correlator to extract the low-lying mass spectrum of the theory.

\section{Cutting all bridges}

In our approach the only bonds which play a role in the evaluation of the observables defining the gauge theory are those belonging to loops. Thus, once a new configuration $G$ is generated, we first get rid of all bridges. One way of achieving this goal is the following.

(1) Eliminate all the dangling ends (see Fig. 2(a) and (b)). At this stage the remaining graph is formed by loops and lines of bridges connecting them.

(2) Build a reduced graph in which the only vertices are the lattice sites with more than two incident occupied bonds. The edges of the graph are formed by lines of occupied bonds (Fig. 2(b)).

(3) Erase one edge at a time and apply each time a cluster reconstruction algorithm (for instance Hoshen-Kopelman [7]) in order to pick off the remaining bridges (Fig. 2(c)). 


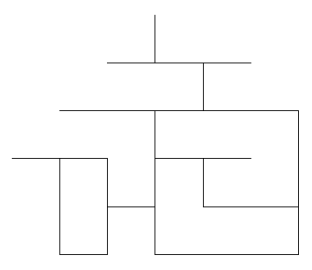

a

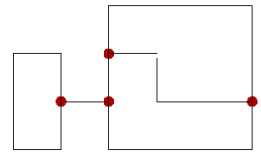

b

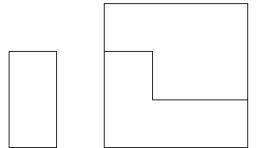

$\mathrm{c}$

Fig. 2. Cutting all bridges of a configuration.

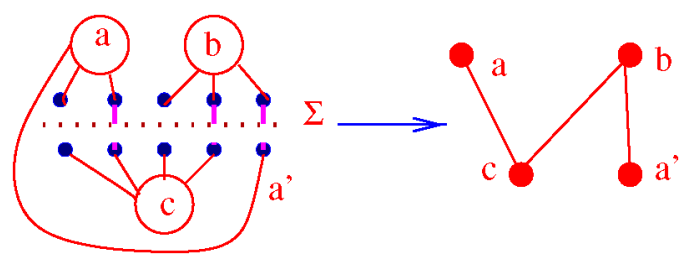

Fig. 3. A 2D sketch of the method used to evaluate the linking of a configuration with a loop $\gamma$. The two lines of solid dots represent the sites on either side of the layer of bonds piercing the surface $\Sigma$ (dotted line). The vertical broken lines are the switched off bonds. The big open circles represent the clusters of the cut graph to which the sites of the layer belong. The cluster $a$ reaches both margins of the layer.

In order to check whether a planar loop $\gamma \subset \tilde{\Lambda}$ is linked to a configuration $G$, we first project out all bridges as discussed. Then we switch off the layer of occupied bonds which pierce the planar surface $\Sigma$ encircled by $\gamma$ and rebuild the cluster structure of the cut graph. For a non-trivial linking there must be at least one cluster which reaches the layer on either side (see the graph on the left of Fig. 3). In such a case we build an auxiliary graph in which the vertices represent the clusters of sites on either side of the layer; a cluster connecting sites of opposite sides is represented by two vertices, one for each side (see the graph on the right of Fig. 3). We draw an edge between two clusters in the opposite sides of the layer if they are connected by switched off bonds. The configuration is truly linked to $\gamma$ if there is a path (at least) joining two vertices lying on opposite margins of the layer, but belonging to the same cluster of the cut graph (like the vertices $a$ and $a^{\prime}$ of the figure).

The whole procedure of cutting all bridges of a configuration and then evaluating its linking property with a set of Wilson loops is time demanding, thus a good implementation is mandatory. However the variance of the measured quantities turns out to be small, hence one can reach more precise results than the corresponding estimates in ordinary gauge theories.

\section{Confinement mechanisms}

According to the recipe given in the previous section, the vacuum expectation value of the Wilson operator $W_{\gamma}$ is defined as 


$$
\left\langle W_{\gamma}\right\rangle=\lim _{N \rightarrow \infty} \sum_{i} W_{\gamma}\left(G_{i}\right) / N=\frac{\text { number of config. unlinked to } \gamma}{\text { total number of configurations }} .
$$

What is the functional form of this quantity for large loops? It depends on the value of the occupation probability $p$. If $p$ is below the percolation threshold $p_{c}$, then there are only finite-size clusters. If the loop $\gamma$ has much a larger size than the clusters, then the configurations where $W_{\gamma}\left(G_{i}\right)=0$ necessarily have some cluster located near the loop. The number of these clusters grows linearly with the perimeter $|\gamma|$ of $\gamma$ and produces the exponential decay $\left\langle W_{\gamma}\right\rangle \propto e^{-\alpha|\gamma|}$. We say that the theory is deconfined. On the contrary if $p>p_{c}$ the theory is confined. Indeed in such a case there is an infinite cluster, therefore the number of closed paths linked to $\gamma$ grows with the area $A$ of the minimal surface $\Sigma$ encircled by $\gamma$. These paths will pierce $\Sigma$ at points; let $N=\alpha A$ be their mean number. Assuming these points to be randomly distributed on the surface, the probability of finding $k$ such points inside $\Sigma$ is binomial,

$$
P_{N}(k)=\left(\begin{array}{c}
N \\
k
\end{array}\right) \alpha^{k}(1-\alpha)^{N-k} .
$$

Note that only the $k=0$ term contributes to the numerator of (4.1), so the expectation value of the Wilson loop becomes

$$
\left\langle W_{\gamma}\right\rangle=(1-\alpha)^{N}=e^{-\sigma A}, \quad \sigma=-\alpha \log (1-\alpha) .
$$

One thus apparently obtains an area law decay with string tension $\sigma$ for any Wilson loop, including those of small size. There is however a flaw in the argument; even if the configurations are obtained by populating each bond (or site) of the lattice independently with a probability $p$, when all the bridges are erased it is no longer true that the remaining bonds are randomly distributed, as anticipated previously. In fact, since the interaction among the intersection points of $\Sigma$ is rather weak, we expect an area law only for large enough Wilson loops.

Strictly speaking, area law and string tension do not seem to have been considered previously in percolation studies, being typical notions of gauge theory. There is however an intimately related quantity, the surface tension, which can also be defined in percolation [8]. Three-dimensional spin systems below critical temperature offer a simple context where this notion can be developed. While in an infinite volume the system shows a spontaneous symmetry breaking, in finite volume this cannot occur, and interfaces appear, separating extended domains of different magnetization. One can define an interface free energy $\mathcal{F}$ in terms of the partition functions of the three-dimensional system with a suitable choice of boundary conditions. If, for instance, $Z_{a}\left(Z_{p}\right)$ is the partition function of an Ising system in a cubic lattice with size $L$ and periodic in the $x$ and $y$ directions and of size $\ell$ and antiperiodic (periodic) in the $z$ direction, one has $\frac{Z_{a}}{Z_{p}}=e^{-\ell \mathcal{F}}$ and it can be shown [9] that in the large $L$ limit one has

$$
\mathcal{F} \propto \sigma L^{2},
$$

$\sigma$ being the surface tension of the interface.

Defining bond percolation as the $q \rightarrow 1$ limit of $q$-state Potts model one can identify $Z_{a}$ as the weighted sum of the configurations where no cluster is wrapped around the $z$ 
direction, while $Z_{p}$ is the total partition function, therefore $Z_{p}=1$. As a consequence, in the percolating region one can use Eq. (4.4) to define the surface tension and it is almost obvious, owing to our definition of Wilson loops, that it coincides with the string tension.

In gauge theories two different confining mechanisms were proposed. One is based on the condensation of center vortices [10]. These objects are string-like structures which are created by gauge transformations with a non-trivial homotopy associated to the center of the gauge group $\mathcal{C}(G)$. In a 3D lattice the center vortices are represented by a skein of loops forming an infinite network in the confining phase [11]. Each center vortex linked with a Wilson loop contributes to it with a factor of $\zeta \in \mathcal{C}(G)$, thus each configuration contributes with a factor $\zeta^{n}$, where $n$ is the number of linked loops. Although at the end the overall effect is again a decay of $\left\langle W_{\gamma}\right\rangle$ with an area law, such a mechanism is slightly different from the one we have described in pure percolation, where a single linked loop suffices to give a weight zero to the configuration. Another difference is that center vortices carry some conserved charge. For instance, if $\mathcal{C}(G)=\mathbb{Z}_{N}$ the vortex flux is conserved modulo $N$, while paths of random percolation do not carry any conserved charge and can intersect freely.

The other confining mechanism is based on the old conjecture [12] that the vacuum behaves like a dual superconductor. The key element of this picture is the monopole condensate which squeezes the gauge field generated by a pair of sources (quarks) into a thin flux tube (the dual version of the Abrikosov vortex). This causes the Wilson loop to decay with an area law. One is led to conjecture that such a thin flux tube should vibrate as a free string [13]. As a consequence, the expectation value of a rectangular Wilson loop of size $R \times T$ is expected to have the following asymptotic functional form in the continuum limit [14]

$$
\langle W(R, T)\rangle=C e^{-p(R+T)-\sigma R T} \sqrt{\frac{\eta(i) \sqrt{R}}{\eta(i T / R)}},
$$

where $C, p$ and $\sigma$ are functions of the coupling constant and $\eta$ is the Dedekind function

$$
\eta(\tau)=q^{1 / 24} \prod_{n=1}^{\infty}\left(1-q^{n}\right), \quad q=e^{2 i \pi \tau} .
$$

The factor under the square root accounts for the universal quantum contributions of the supposedly string-like flux tube describing the interaction between far-away sources.

Random percolation, lacking any non-trivial conserved charge, can hardly account for effects which play the role of magnetic monopole condensation. Notwithstanding this difficulty, we get indirect evidence of the formation of a vibrating string-like flux tube by measuring the universal shape effects it produces, as discussed in Section 5.

\section{String tension}

We estimated the string tension $\sigma$ by fitting the mean values of the Wilson loops associated to squares of side $R$ to Eq. (4.5), that in such a case becomes

$$
W(R)=C R^{1 / 4} \exp \left(-2 p R-\sigma R^{2}\right) .
$$




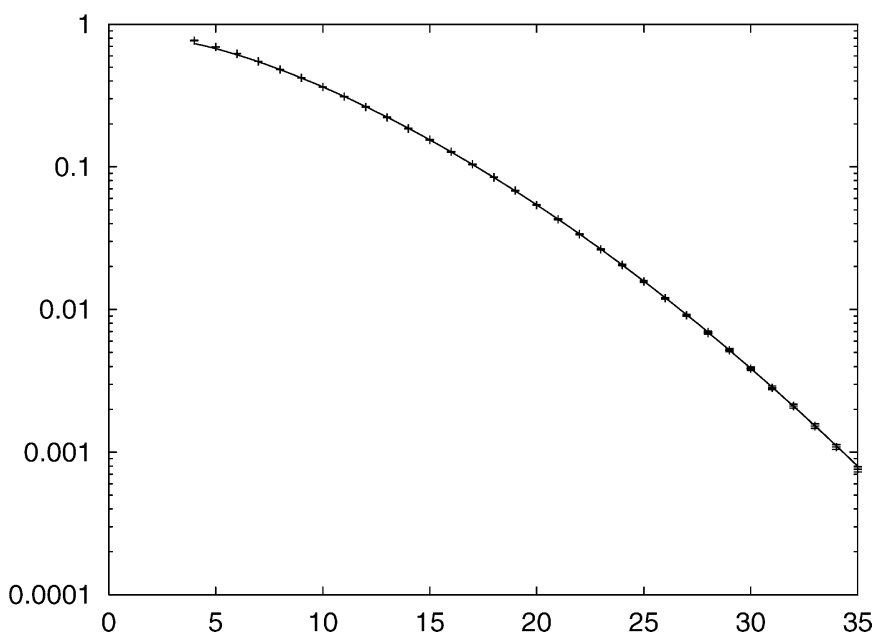

Fig. 4. Square Wilson loop as a function of $R$ for bond percolation in a cubic lattice of size $L^{3}=64^{3}$ at $p=0.26$.

Typically, in a lattice of size $L^{3}$ we considered all the squares with $R \leqslant L / 2$. The fits for not too small $R$ are very good (see Fig. 4), nevertheless the parameters slightly depend on the value $R_{\min }$ of the smallest square included in the fit. Since these formulae are expected to be valid only asymptotically for large values of $R$, we progressively eliminated the data of lower $R$ until stable parameters were obtained.

In order to check for presence of universal shape effects ascribed to the quantum fluctuations of the effective string, we considered, as in [15], the quantity

$$
\mathcal{R}(n, R) \equiv e^{-n^{2} \sigma} \frac{W(R+n, R-n)}{W(R)},
$$

which asymptotically (i.e., large $R$ and $R-n$ ) should be only a function of the ratio $t=\frac{n}{R}$, namely,

$$
\mathcal{R}(n, R) \rightarrow f(t)=\sqrt{\frac{\eta(i) \sqrt{1-t}}{\eta\left(i \frac{1+t}{1-t}\right)}} .
$$

Note that it does not contain any adjustable parameters. This function is plotted in Fig. 5 and compared with the numerical data for three different values of $p$. The presence of the expected universal shape effects seems uncontroversial.

The string tension $\sigma$ is a physical quantity with the dimensions of an inverse square length, hence it is expected to exhibit the following power law behaviour sufficiently close to the percolation threshold

$$
\sigma=S\left(p-p_{c}\right)^{2 v}
$$




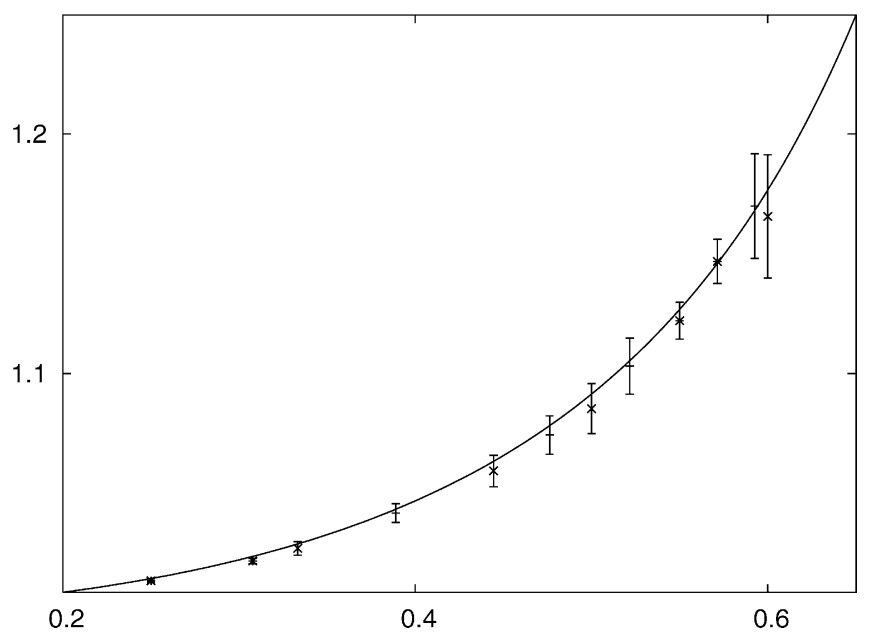

Fig. 5. The universal shape effects of Eq. (5.3) are compared with numerical data of Eq. (5.2) for three different values of $p$.

where $v$ is the critical exponent of the correlation length in $3 \mathrm{D}$ percolation. We used the value $v=0.8765(16)(2)$ of Ref. [16]. ${ }^{1}$

Keeping $v$ as a parameter to be fitted in Eq. (5.4), our result from the data referring to site percolation is $v=0.874(5)$, in agreement with the result quoted from Ref. [16]; similarly, the result we obtain in the bond percolation case is $v=0.859(15) .^{2}$

The percolation threshold $p_{c}$ depends on the lattice and on the kind of percolation (site or bond) which is studied. We checked Eq. (5.4) in the percolating region of three different lattices: simple cubic (SC) with site or bond percolation and body-centered cubic (BCC) with bond percolation. Precise estimates of $p_{c}$ are known and are reported in Table 1. In the SC cases we used a lattice of size $L^{3}=64^{3}$, while in the BCC case we had $L^{3}=55^{3}$. In all cases the one-parameter fit to Eq. (5.4) is very good and the scaling window seems rather wide (see Fig. 6). The resulting amplitudes $S$ for the three lattices are reported in Table 1.

Noteworthy, in order to extract the string tension $\sigma$ we have thus far assumed that the square Wilson loops obey the asymptotic form (5.1), where the factor $R^{\frac{1}{4}}$ accounts for the contribution of the string fluctuations. If one neglected this factor and only took the area term into account, the $\chi^{2}$ test of the critical power law (5.4) would grow worse by one or two orders of magnitude, depending on the kind of lattice. We consider this fact another strong evidence of a vibrating, confining string.

\footnotetext{
1 The first number between parenthesis is the statistical error, the second comes from the uncertainty in the scaling correction exponent $\omega$.

2 The data analysis in the bond percolation case is slightly more complicated, due to the fact that the correlation length is larger, and this induces larger finite size effects as the critical value is approached. In our analysis, we kept this aspect into account.
} 


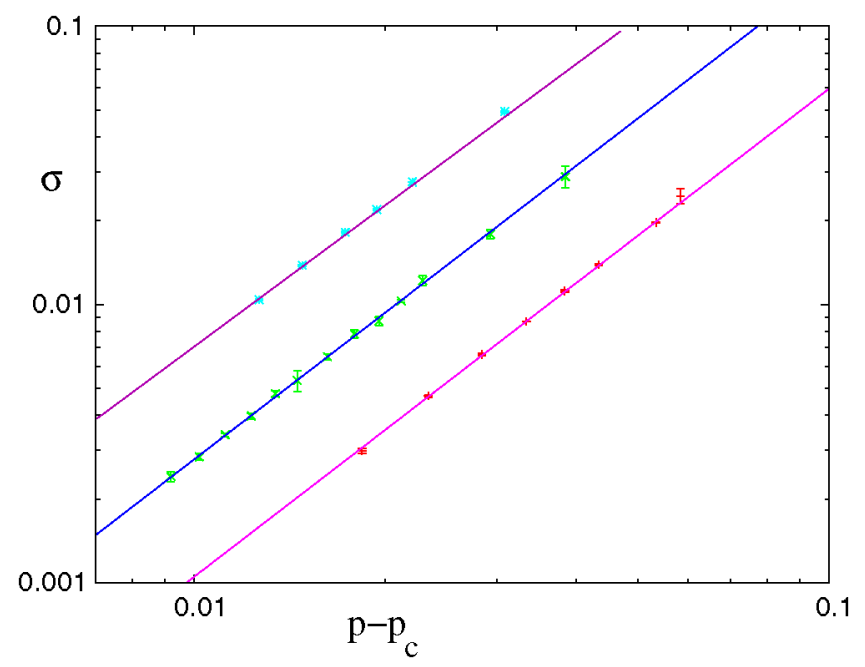

Fig. 6. The string tension $\sigma$ as a function of $p-p_{c}$ for three different lattices: BCC bond (top line) SC bond (middle line) and SC site. The three parallel lines are one-parameter fits to Eq. (5.4). The corresponding amplitudes are reported in Table 1.

Table 1

The amplitude of the string tension for three different lattices. Errors in parenthesis affect the last digits

\begin{tabular}{llrl}
\hline Lattice & $p_{c}$ & $S$ & $\chi^{2} /$ d.o.f. \\
\hline SC site & $0.3116081(7)(2)[16]^{1}$ & $3.370(8)$ & 1.15 \\
SC bond & $0.2488126(5)[17]$ & $8.90(3)$ & 0.30 \\
BCC bond & $0.1802875(10)[17]$ & $22.07(2)$ & 0.98 \\
\hline
\end{tabular}

\section{Spectrum}

As for standard gauge theories, we expect that the confining phase of percolation possesses a rich spectrum of physical states with increasing mass and spin, that we still call glueballs.

The basic method that goes into the computation of such mass spectrum is very simple. One first constructs a linear combination of Wilson loops on a fixed time slice of the threedimensional lattice carrying the quantum numbers of the state one wishes to investigate. One then builds zero momentum operators by summing such a linear combination over the entire spatial lattice. The simplest example of a zero momentum operator coupling to the spin $0^{+}$states is given by

$$
\Phi^{0+}(t)=\sum_{x, y}\left[W_{\square 1}(x, y, t)+W_{\square 2}(x, y, t)\right],
$$

where $W_{\square j}(\vec{x})$ denotes an elementary plaquette variable with base at $\vec{x}=(x, y, t)$ and orthogonal to the $j$ coordinate axis. According to Section 2, in random percolation the plaquette variable is replaced by the dual link variable $\ell_{j}(\vec{x})$, defined as equal to 1 if the corresponding bond belongs to the subset $B_{G}$ of loops and null otherwise. The low-lying 


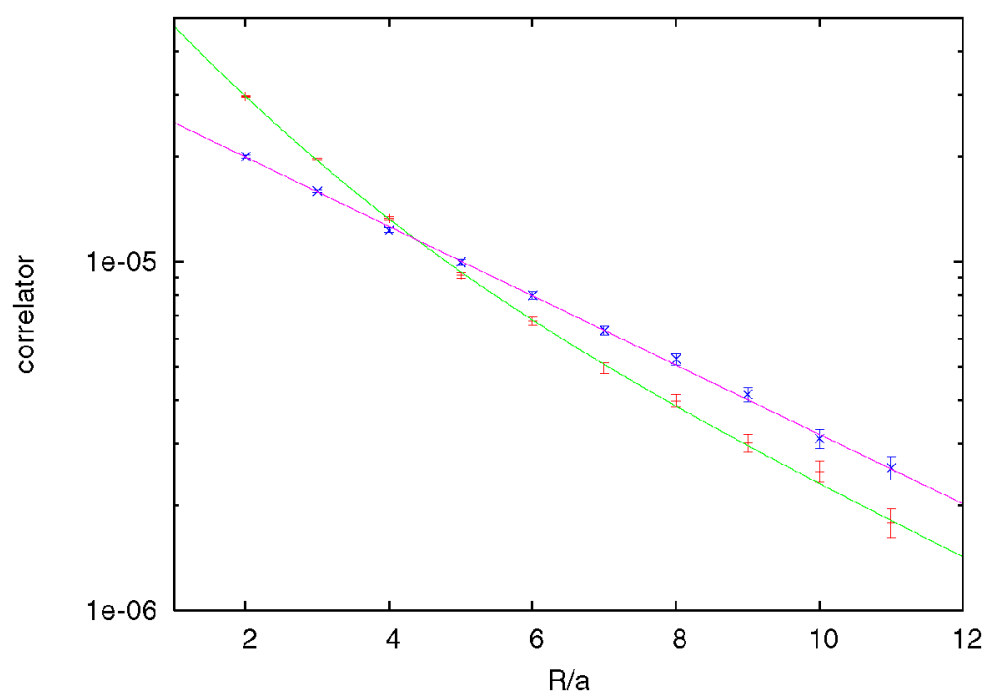

Fig. 7. Comparison between the standard cluster correlator defined in Eq. (6.5) and the connected plaquette-plaquette correlator in a $32 \times 32 \times 64$ cubic lattice at $p=0.260$. The straight line is an exponential fit to the cluster correlator data $(x)$. These data have been displaced downwards by two orders of magnitude for clarity. The other line is a two-exponential fit to the plaquette data $(+)$. The semi-logarithmic plot makes it evident that in the latter case a single exponential does not suffice.

mass spectrum can be extracted by studying the exponential decay of the connected correlator $C(t)=\left\langle\Phi^{0+}(t) \Phi^{0+}(0)\right\rangle-\left\langle\Phi^{0+}\right\rangle^{2}$, which is expected to have the following asymptotic expansion

$$
C(t)=\sum_{n} c_{n} e^{-m_{n} t}
$$

where $c_{n}$ denote positive constants and $m_{n}$ are the glueball masses. An example of such a correlator in a SC bond lattice is reported in Fig. 7, where it is evident that at least two different scalar states contribute to $C(t)$. The estimates of the lowest mass in the range $0.258 \leqslant p \leqslant 0.270$ fit well with the scaling form (see Fig. 8)

$$
m=M_{0}\left(p-p_{c}\right)^{v},
$$

with

$$
M_{0}=12.45 \pm 0.07 \text {. }
$$

Also the first excited state seems to follow the same power law as expected, though the errors are rather big. Its mass $M_{0}^{\prime}$ is about twice $M_{0}$.

Such a behaviour is very different from the one observed in the standard two-point correlation function of the percolation problem, defined as the probability $G\left(\vec{x}, \vec{x}^{\prime}\right)$ that the sites $\vec{x}$ and $\vec{x}^{\prime}$ are in the same cluster. ${ }^{3}$ The corresponding connected, zero-momentum,

3 This quantity is directly related to the correlator in $q \rightarrow 1$ limit of the $q$-state Potts model, see for instance Ref. [18], p. 156. 


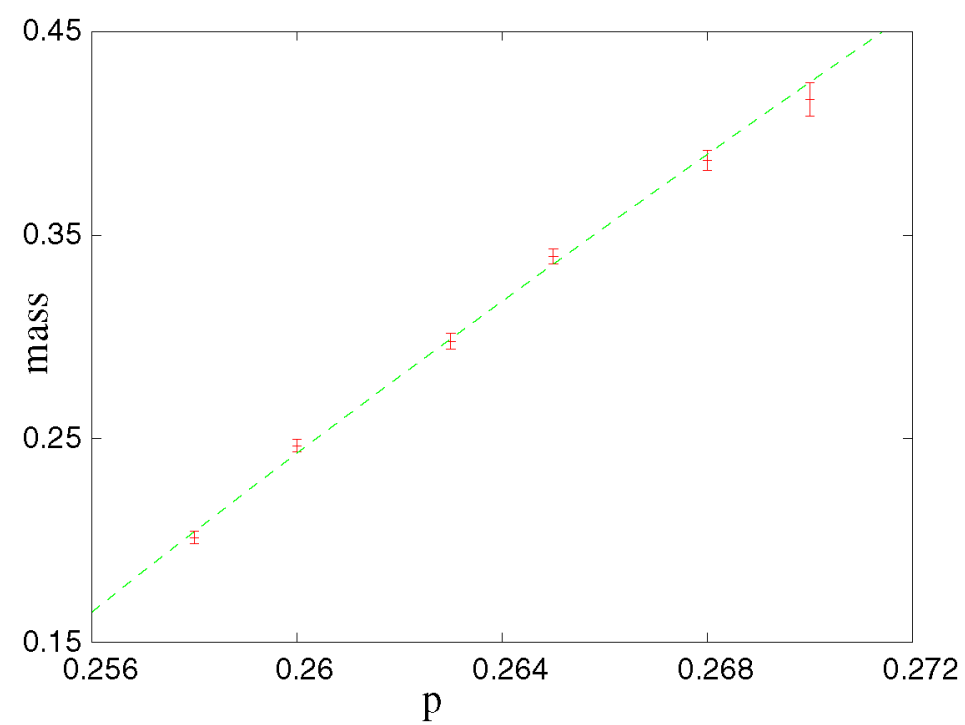

Fig. 8. The mass of the lowest state as a function of $p$ in a simple cubic bond percolating lattice. The dashed curve is a one-parameter fit to Eq. (6.3).

projection

$$
\mathcal{C}(t)=\sum_{x, y}[G(0, \vec{x})-G(0, \infty)]
$$

exhibits a single exponential behaviour with a mass term which coincides, within the numerical accuracy, with that of the lowest energy state coupled to the plaquette operator (see Fig. 7). This indicates that the standard cluster correlator couples only to the lowest glueball, while the new observables suggested by the gauge theory interpretation of the percolation disclose a totally unexpected spectrum of physical states.

The lightest spinning glueball is the $2^{+}$state. It can be observed in the exponential decay of the correlation function of the operator

$$
\Phi^{2+}(t)=\sum_{x, y}\left[\ell_{1}(x, y, t)-\ell_{2}(x, y, t)\right] .
$$

A difficulty encountered in this case is that the signal is drowned within the statistical noise for values of $t$ beyond three or four lattice spacings. In spite of this accuracy problem, one can still verify that in a SC bond lattice the scaling form (6.3) is approximately obeyed with an amplitude

$$
M_{2^{+}}=80 \pm 10
$$

In order to have more accurate results on the mass spectrum the basis of the operators should be enlarged to Wilson loops of different shapes, trying to enhance their overlap with the glueball states. 


\section{Deconfinement at finite temperature}

In quantum field theory the concept of temperature is introduced by simply compactifying the Euclidean time direction and identifying the inverse temperature with the temporal extension of the space-time manifold. Lattice field theories at a temperature $T$ are formulated in a slab which is infinite in the spatial dimensions, but of finite length $\ell=1 / T$ (in lattice spacing units) and periodic in the remaining temporal direction.

In any confining gauge theory there is a critical temperature $T_{c}$ above which the system is deconfined in the sense that for $T \geqslant T_{c}$ the string tension $\sigma$ vanishes. In this section we demonstrate that the same phenomenon also occurs in random percolation. In the latter case the deconfinement mechanism is particularly transparent, showing its purely geometric origin: non-vanishing string tension requires an infinite, percolating, cluster. Shrinking the width of the slab reduces the number of possible percolating paths along the spatial directions until the infinite cluster crumbles away, yielding a vanishing $\sigma$.

To put it in different terms, note that as the temperature varies from zero to infinity a three-dimensional system is gradually dominated by two-dimensional behaviour; in particular the percolation threshold is a decreasing function of the space dimensions. For instance in the SC bond lattice at $T=0$ the percolation threshold is at $p_{c}(D=3)=0.2488 \ldots$ (see Table 1). At $T=\infty$ the system reduces to a square lattice, where $p_{c}(D=2)=\frac{1}{2}$, hence heating a system which at zero temperature lies in the percolating phase with $p<\frac{1}{2}$ inevitably undergoes a deconfinement transition at a finite temperature $T_{c}$.

In order to estimate $T_{c}$ in various site or bond percolation lattices we considered a slab of size $L_{x} \times L_{y} \times \ell$ with $L_{x}=L_{y}=L \gg \ell$ and periodic boundary conditions in all directions (see Fig. 9). We calculated the probability $R_{L}(p)$ for a cluster to wrap around one of the large dimensions. Wrapping probabilities can be defined in different ways: wrapping around the $x$ direction, around the $y$ direction, around either direction, around both

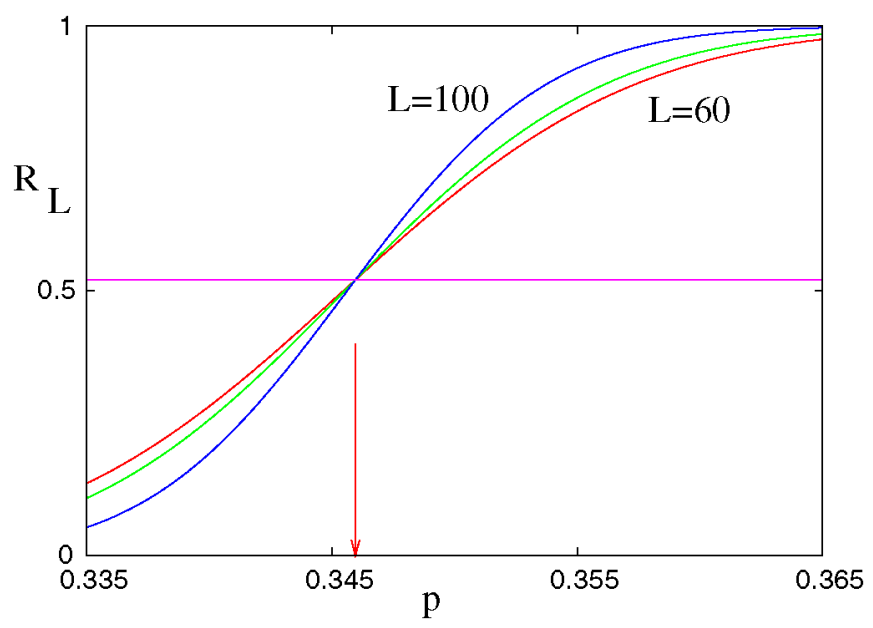

Fig. 9. The wrapping probability $R_{L}$ in a simple cubic site percolating lattice for $\ell=7$ and $L=60,70,100$. The vertical arrow denotes the estimated value of $p_{\ell}$ at $L=\infty$ and the horizontal line indicates the exact planar value of $R_{\infty}$. 
directions, etc. To be definite, we consider wrapping around the $x$ direction. For large $L$ it coincides with the probability that the system percolates along $x$.

We evaluated $R_{L}(p)$ using a very efficient algorithm described by Newman and Ziff [19]. For a bond percolation problem it consists of repeatedly adding a random bond to an initially empty lattice, identifying the clusters joined by the bond and merging them if they are different. At each step one checks whether the touched cluster wraps around $x$ using a clever method described in Ref. [20]. The process stops as soon as a wrapped cluster is detected. In this way one can evaluate the probability $Q_{L}(n)$ that a random configuration with $n$ occupied bonds is wrapping around $L_{x}$ for any $n \leqslant N$, where $N$ is the total number of bonds of the lattice. This method may be adapted to site percolation in a straightforward way. Then one simply finds the required quantity $R_{L}(p)$ for any value of $p$ by convolution with the binomial distribution

$$
R_{L}(p)=\sum_{n}\left(\begin{array}{c}
N \\
n
\end{array}\right) p^{n}(1-p)^{N-n} Q_{L}(n) .
$$

Fig. 9 shows some examples of $R_{L}(p)$ in the case of SC site percolating lattice. In the $L \rightarrow \infty$ limit this wrapping probability becomes a step function. We have

$$
R_{\infty}(p)= \begin{cases}0 & \text { for } p<p_{\ell} \\ 1 & \text { for } p>p_{\ell}\end{cases}
$$

where the threshold value $p=p_{\ell}$ depends on the type of lattice and on its width $\ell$. When $L=\infty$ the slab system is equivalent to a $2 \mathrm{D}$ torus, where the wrapping probability at criticality has been calculated exactly by Pinson [21] and is $R_{\infty}\left(p_{\ell}\right)=0.521058290 \ldots$ We can use his result to measure the value of $p_{\ell}$ in a slab of width $\ell$ by finding the value of $p$ for which

$$
R_{L}(p)=R_{\infty}\left(p_{\ell}\right) .
$$

This method was first applied in the case of the Ising model in Ref. [22] (with the appropriate value of $R_{\infty}$, of course). These estimates in the case of 2D percolation turn out to scale particularly well with the system size: Newman and Ziff argued that in planar lattices the leading order finite size correction goes like

$$
p=p_{\ell}+c L^{-2-1 / \nu_{2}}=p_{\ell}+c L^{-11 / 4},
$$

where $v_{2}=\frac{4}{3}$ is the thermal exponent of $2 \mathrm{D}$ random percolation.

We checked it in the slab geometry finding good agreement for $L$ large enough, as Fig. 10 shows in the case of a slab of width $\ell=6$.

We estimated in this way the threshold $p_{\ell}$ for seven different lattices. The results are reported in Table 2. The slab widths (or equivalently the inverse temperatures) were chosen in such a way that the values of $p_{\ell}$ lie in the scaling region of the string tension $\sigma$, as determined in Section 5. In this manner we were able to evaluate also the ratio $T_{c} / \sqrt{\sigma}$ using the amplitudes of Table 1. It turns out that these ratios for different lattices and widths are clearly compatible with a common value, as required by universality (see last column of Table 2).

In $(d+1)$-dimensional field theory at finite temperature there is a characteristic interplay between $d+1$ and $d$ critical behaviours. This is particularly evident in the present 


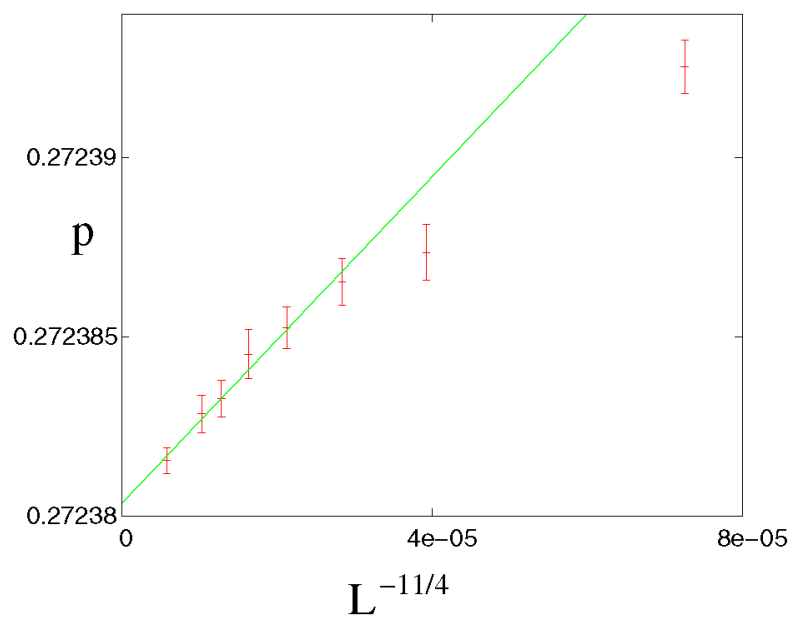

Fig. 10. Finite-size scaling of the estimated $L \rightarrow \infty$ value of $p_{\ell}$ for bond percolation in a slice of SC lattice of size $L \times L \times 6$ with $32 \leqslant L \leqslant 80$. Each point corresponds to about $10^{8}$ configurations. The solid line is a fit to Eq. (7.4).

\section{Table 2}

The critical $p_{\ell}$ for different lattices at different temperatures and the corresponding universal ratio $T_{c} / \sqrt{\sigma}$ as obtained by combining Eqs. (5.4) and (7.5). Errors in parenthesis affect the last digits

\begin{tabular}{llll}
\hline Lattice & $1 / T$ & $p_{\ell}$ & $T_{c} / \sqrt{\sigma}$ \\
\hline SC site & 7 & $0.3459514(12)$ & $1.494(11)$ \\
BCC bond & 3 & $0.21113018(38)$ & $1.497(10)$ \\
BCC bond & 4 & $0.20235168(59)$ & $1.506(11)$ \\
SC bond & 5 & $0.278102(5)$ & $1.480(12)$ \\
SC bond & 6 & $0.272380(2)$ & $1.492(13)$ \\
SC bond & 7 & $0.268459(1)$ & $1.500(13)$ \\
SC bond & 8 & $0.265615(5)$ & $1.504(14)$ \\
\hline
\end{tabular}

instance: the $p_{\ell}$ values are extracted through a 2D percolation power law (7.4) in order to take into account the finite size scaling tied to $L$. However the tower of $p_{\ell}$ values as a function of the width $\ell$ obeys a typical power law of 3D percolation:

$$
p_{\ell}=p_{c}+\frac{1}{\left(\mathcal{T}_{c} \ell\right)^{1 / v}},
$$

where $v$ indicates, as in all the other formulae of this paper, the thermal exponent of 3D percolation, $p_{c}$ is the critical threshold as listed in Table 1 and the amplitude $\mathcal{T}_{c}$ depends on the kind of lattice. Fig. 11 shows the four $p_{\ell}$ values of the bond SC lattice as a function of $\ell^{-1 / v}$. A one-parameter fit to Eq. (7.5) yields

$$
\mathcal{T}_{c}=4.45 \pm 0.02 \text {. }
$$

It should be noted parenthetically that, as the $p_{\ell}$ 's are essentially two-dimensional quantities, they can be evaluated with high precision. Comparison between Tables 1 and 2 shows that in some cases the level of precision of those overcomes that of the best estimates of $p_{c}$. 


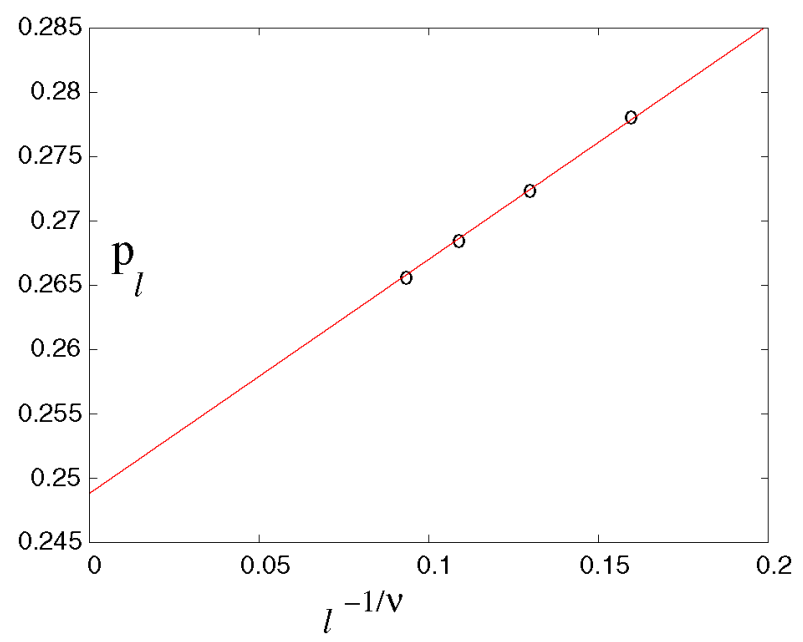

Fig. 11. Finite-size scaling of $p_{\ell}$ as a function of $\ell^{-1 / v}$, where $\ell$ is the width of a SC lattice in bond percolation. The solid line is a one-parameter fit to Eq. (7.5). The statistical errors are much smaller than the symbol size.

Moreover, with a modest additional computational effort, it would be possible to further improve their precision. Thus one could perhaps envisage to apply systematically Eq. (7.5) to improve the estimates of $p_{c}$ and/or $v$.

\section{Universality class of deconfinement}

The deconfined phase of any $(d+1)$-dimensional gauge theory at finite temperature is characterized by the vanishing of the string tension $\sigma$. The interaction between static sources (quarks) is described in terms of Polyakov operators. These are straight Wilson loops wrapped around the short periodic direction $\ell$ (see Fig. 12). The Polyakov-Polyakov correlator $\langle P(0) P(\vec{R})\rangle$ of two parallel Polyakov operators only depends on their relative positions in the $d$-dimensional sub-lattice. At the deconfining point it is expected to obey a power law dictated by the universality class of the transition.

The critical behaviour of gauge theories at the deconfining temperature is well described by the Svetitsky-Yaffe (SY) conjecture [23] which can be formulated as follows. Suppose a $(d+1)$-dimensional gauge theory with gauge group $G$ has a second-order deconfinement transition at a certain temperature $T_{c}$; then its universality class is the same of the order-disorder transition of a $d$-dimensional spin system with a global symmetry group coinciding with the center of the gauge group. In particular, the Polyakov-Polyakov correlator corresponds to the spin-spin correlator of such a $d$-dimensional system. Therefore, at the critical point, it should decay as

$$
\langle P(0) P(\vec{R})\rangle=\frac{\text { const }}{R^{d-2+\eta}},
$$

where $\eta$ is the magnetic exponent of the spin model. The validity of this conjecture has been well established in a large number of numerical studies. 


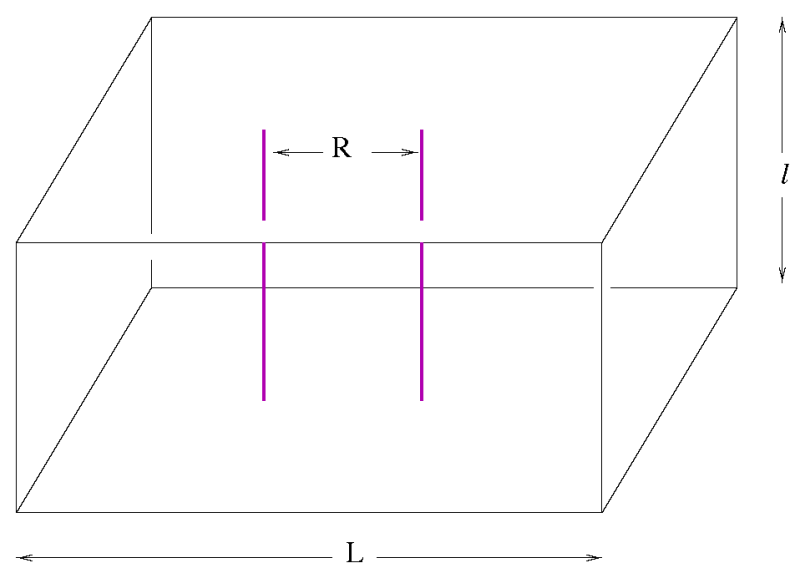

Fig. 12. The slab geometry of the finite temperature setting. The two thick lines represent two Polyakov loops wrapping in the periodic temperature direction.
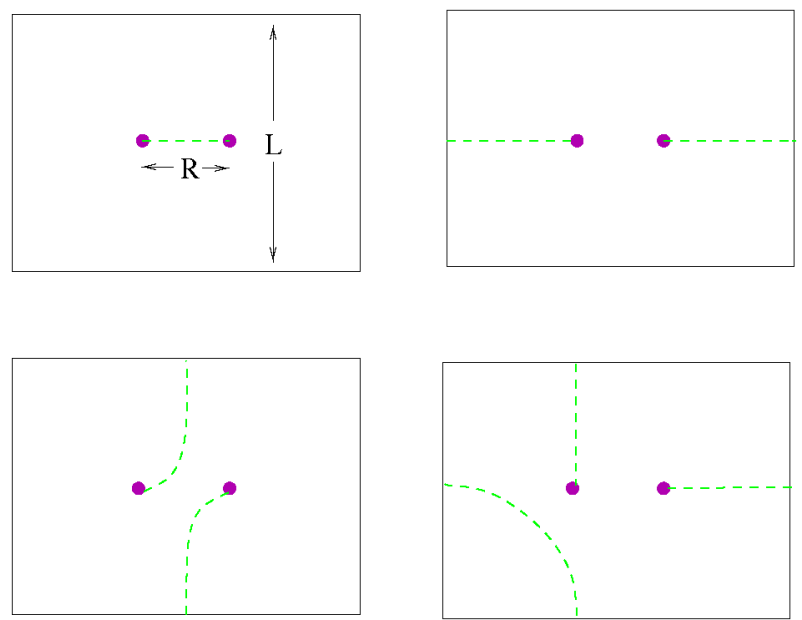

Fig. 13. The dashed lines represent the 1D section of the four topologically different surfaces bounded by the two Polyakov loops (solid circles) in the case of periodic bc.

In the present case the above conjecture requires some generalization, owing to the fact that not only the center, but the whole gauge group is trivial. Actually the SY conjecture is somehow related to the dimensional crossover in a layered lattice system [24]. The universality class of such a system, as it approaches a critical point, depends on the number of spatial directions which are going to infinity in the thermodynamic limit. This simple observation provides the basis for arguing that the critical behaviour of percolation in a slab of finite thickness is well described by the $2 \mathrm{D}$ percolation universality class. This is also supported by the fact that finite size scaling of threshold probability $p_{\ell}$ is driven by the thermal exponent $v_{2}$, as Eq. (7.4) and Fig. 10 show. 


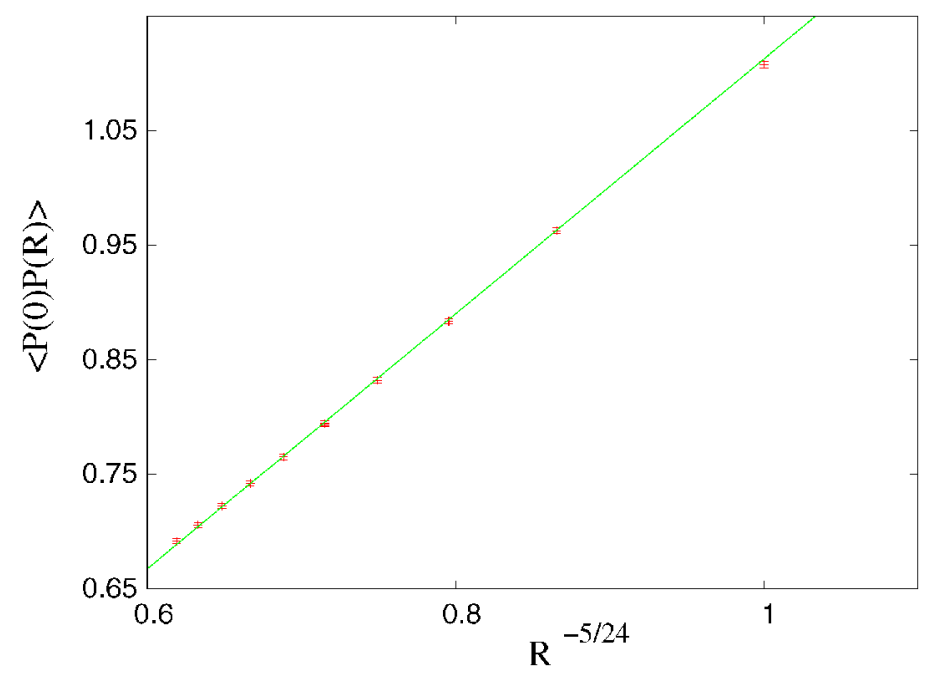

Fig. 14. Polyakov-Polyakov correlator at deconfining temperature as a function of the scaling variable $R^{-\eta}$, where $\eta=\frac{5}{24}$ is the magnetic exponent of 2D percolation. The lattice size is $200 \times 200 \times 6$. The solid line is a one-parameter fit to Eq. (8.1) of the data with $R>2$. The resulting $\chi^{2} /$ d.o.f. is 0.46 .

Such a conclusion is much less obvious, and more interesting, when considering the Polyakov-Polyakov correlator. This quantity can be evaluated using exactly the same method described in Section 3 for the Wilson loops. One simply has to take into account that in the Wilson loop the surface $\Sigma$ (see Fig. 3) used to evaluate cluster wrapping is a rectangle, while in the latter case is a cylinder bounded by the two Polyakov lines. Periodic boundary conditions put into play another difference: there are now four topologically different surfaces bounded by the two Polyakov lines (see Fig. 13), hence the correlator must be written as the sum of these four contributions. When the distance $R$ between Polyakov lines is much smaller than lattice size $L$ the main contribution comes from the top two surfaces of Fig. 13.

One instance is reported in Fig. 14, where we plot the estimated Polyakov-Polyakov correlator extracted from $10^{4}$ configurations in a $200 \times 200 \times 6$ lattice at the critical value of $p_{6}$ as determined in Table 2. Plotting these data versus $R^{-\eta}$ with $\eta=\frac{5}{24}$ shows linear behaviour, as expected for a critical system which lies in the universality class of $2 \mathrm{D}$ percolation.

\section{Conclusions}

We have studied some consequences of a new point of view in three-dimensional random percolation which allows us to reinterpret it as a full-fledged gauge theory.

A key difference between the conventional and the present approach is that instead of studying the universal scalings through the size distribution of random clusters we only consider their topological entanglement with suitable closed paths. This suggests a reformulation in the language of gauge theory. In this context a more detailed description of 3D 
percolation universality class emerges as a major issue: the gauge-percolation dictionary leads to define new physical quantities that can be used to extract new universal amplitude ratios.

A typical example is the ratio $T_{c}^{2} / \sigma$ between the (square of) deconfining temperature $T_{c}$ and the string tension $\sigma$. We evaluated it on seven different lattices (see Table 2) finding excellent agreement with universality. Notice that neither $T_{c}$ nor $\sigma$ are truly foreign concepts of percolation theory: slab percolation has always been a subject of intensive study. We have seen that string tension is strictly related to the surface tension, which is also defined in percolation [8]. The novelty introduced by the gauge theory interpretation is a previously unsuspected relationship between slab percolation and surface tension.

Another class of universal amplitude ratios came to us as a surprise. It turns out that the Wilson loop correlators receive contribution from a tower of physical states of increasing mass and spin, like in ordinary gauge theories. The ratios of their masses define universal quantities which further characterize the universality class of 3D percolation.

Transcribing percolation into gauge theory language has also some interesting consequences in the study of quark confinement mechanisms. The confinement generated by a random percolating cluster is similar, but not identical, to that produced by an infinite network of center vortices. In particular the latter carries some conserved charge which regulates their mutual intersections, while the former does not carry any conserved charge and intersects freely. This indicates that the intersection rules of center vortices do not play an important role in producing confinement.

One of the most surprising findings of the present approach is the observation of some universal shape effects in the vacuum expectation value of Wilson loops which have been always ascribed to a different picture of confinement. This picture says that the flux of the gauge field generated by pair of quarks is squeezed by the magnetic monopole condensate into a string-like structure which can vibrate freely. It turns out that these very vibrations generate the universal effects mentioned above. Apparently, the two different pictures of confinement are different descriptions of the same physical phenomenon.

It would be interesting to extend our percolation approach to a $4 \mathrm{D}$ gauge theory. This would require the study of plaquette percolation and the Wilson loops should measure their entanglement with closed surfaces.

\section{Acknowledgements}

M.P. acknowledges support received from Enterprise Ireland under the Basic Research Programme.

\section{References}

[1] See, e.g., D. Stauffer, A. Aharony, Introduction to Percolation Theory, second ed., Taylor \& Francis, London, 1994.

[2] F. Gliozzi, M. Panero, A. Rago, Nucl. Phys. B (Proc. Suppl.) 129 (2004) 736, hep-lat/0309061.

[3] C.M. Fortuin, P.W. Kasteleyn, Physica (Amsterdam) 57 (1972) 536.

[4] H.A. Kramers, G.H. Wannier, Phys. Rev. 60 (1941) 252. 
[5] F. Gliozzi, S. Vinti, Nucl. Phys. B (Proc. Suppl.) 53 (1997) 593, hep-lat/9609026.

[6] F. Gliozzi, Phys. Rev. E 66 (2002) 016115, cond-mat/0201285.

[7] J. Hoshen, R. Kopelman, Phys. Rev. B 14 (1976) 3438.

[8] C.K. Harris, J. Phys. A: Math. Gen. 18 (1985) 2259.

[9] M.E. Fisher, V. Privman, J. Stat. Phys. 33 (1983) 74.

[10] G. 't Hooft, Nucl. Phys. B 138 (1978) 1.

[11] M. Engelhardt, K. Langfeld, H. Reinhardt, O. Tennert, Phys. Rev. D 61 (2000) 054504, hep-lat/9904004.

[12] S. Mandelstam, Phys. Lett. B 53 (1975) 476;

S. Mandelstam, Phys. Rep. C 23 (1976) 245;

Y. Nambu, Phys. Rev. D 10 (1974) 4262.

[13] M. Lüscher, K. Symanzik, P. Weisz, Nucl. Phys. B 173 (1980) 365.

[14] J. Ambiørn, P. Olesen, C. Peterson, Nucl. Phys. B 244 (1984) 262.

[15] M. Caselle, R. Fiore, F. Gliozzi, M. Hasenbusch, P. Provero, Nucl. Phys. B 486 (1997) 245, hep-lat/9609041.

[16] H.G. Ballesteros, L.A. Fernandez, L.A.V. Martin-Mayor, A. Munoz-Sudupe, G. Parisi, J.J. Ruiz-Lorenzo, J. Phys. A 32 (1999) 1.

[17] Ch.D. Lorenz, R.M. Ziff, Phys. Rev. E 57 (1998) 230.

[18] J. Cardy, Scaling and Renormalization in Statistical Physics, Cambridge Univ. Press, Cambridge, 1996.

[19] M.E.J. Newman, R.M. Ziff, Phys. Rev. Lett. 85 (2000) 4104.

[20] J. Machta, Y.S. Choi, A. Lucke, T. Schweizer, L.M. Chayes, Phys. Rev. E (1996) 1332.

[21] H.T. Pinson, J. Stat. Phys. 75 (1994) 1167.

[22] M. Caselle, M. Hasenbusch, Nucl. Phys. B 470 (1996) 435, hep-lat/9511015.

[23] B. Svetitsky, L. Yaffe, Nucl. Phys. B 210 (1982) 423.

[24] T.W. Capehart, M.E. Fisher, Phys. Rev. B 13 (1976) 5021. 